\title{
Soft sensing for two-phase flow using an ensemble Kalman filter
}

\author{
A. Gryzlov*, M. Leskens**, R.F. Mudde* \\ * Department of Multi-Scale Physics, Delft University of Technology, Delft, 2628 BW, \\ the Netherlands (Tel: 31(0)152783210; e-mail:a.gryzlov@tudelft.nl) \\ ** Department of Process Modelling and Control, TNO Science and Industry, \\ Eindhoven, the Netherlands (e-mail: martijn.leskens@tno.nl)
}

\begin{abstract}
A new approach for real-time monitoring of horizontal wells, which is based on data assimilation concepts, is presented. Such methodology can be used when the direct measurement of multiphase flow rates is unfeasible or even unavailable. The real-time estimator proposed is an ensemble Kalman filter employing a dynamic model of the pipe flow and information from several downhole pressure sensors with a single measurement of the flow velocity and composition. By means of simulation examples it is shown that the proposed algorithm operates quite accurately both for noisy synthetic measurements and artificial data generated by the OLGA simulator.
\end{abstract}

Keywords: Distributed state estimation, Ensemble Kalman filter, Two-phase flow, Inverse dynamic problem

\section{INTRODUCTION}

The growing demand for hydrocarbon production has resulted into improved oilfield management with various monitoring and optimization strategies (Glandt, 2003, Jansen et al., 2008). These strategies in turn strongly rely on the efficiency of downhole equipment which is used to obtain real-time oil and gas production rates with sufficient spatial and temporal resolution. In particular, multiphase flowmeters installed downhole can improve the production of long horizontal wells by allocating the zones of oil, gas and water inflow. However, existing multiphase meters are expensive, inaccurate or accurate only within a limited operating range and therefore such monitoring is unrealistic.

To overcome these problems one can use so-called multiphase soft-sensors, i.e. to estimate flow rates from conventional meters, such as downhole pressure gauges, in combination with a dynamic multiphase flow model. Despite the variety of soft-sensing techniques (which are also referred to as data assimilation methods), one can note two principal approaches. Variational data assimilation, which is based on the minimization of a cost function within a certain time interval, and sequential methods or filtering when the state of the system is updated every time instant data becomes available. One way to solve these sequential data assimilation problems is to use Kalman filtering (Kalman, 1960). This method, which was originally developed for linear models, has got numerous extensions (Jazwinski, 1970, Evensen, 1994 and Julier et al., 2000) to deal with non-linearity, which is the case for most industrial processes.

Although there are numerous applications of soft-sensing techniques in oil and gas industry, they mainly deal with the estimation of reservoir properties (Naevdal et al., 2003, Evensen et al., 2007). The range of wellbore flow application includes gas-lift wells (Bloemen et al., 2004) and underbalanced drilling (Lorentzen et al., 2001). Also, the Kalman filter has been used for tuning the parameters of two phase flow models (Lorentzen et al., 2003). Leskens et al. (2008) considered the simultaneous estimation of downhole oil, water and gas flow rates from downhole pressure and temperature measurements in a single well. This approach has been extended by de Kruif et al. (2008) to the multilateral well case both for the two-phase (oil and gas) and three-phase (oil, gas and water) cases.

Despite the variety of applications considered, little attention has been given to the inflow allocation problem. More specific, long horizontal wells with a continuous inflow profile from a reservoir to a wellbore require the use of softsensing techniques for the gas breakthrough prediction.

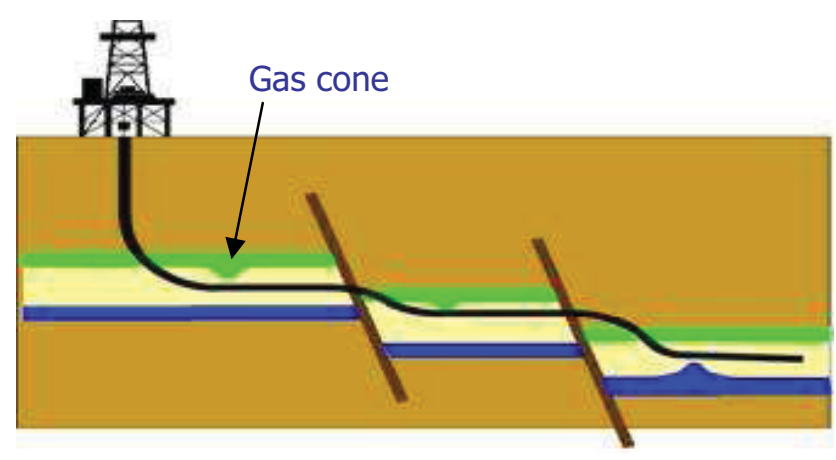

Fig. 1. Schematic view of a horizontal well.

Gas coning is a phenomenon where the gas-oil contact of a reservoir moves towards a producing well (see Figure 1). At a certain moment the gas-oil contact will reach the well and gas breakthrough can happen causing a large gas influx. Consequently, the gas phase may start to dominate production making the well uneconomical. In order to handle or prevent this, several strategies are available. However, the most convenient countermeasure is to isolate gas producing 
zones of a wellbore by means of inflow control valves. The purpose of a soft-sensor is to provide the inflow control valves with information of the downhole flow rate distribution.

This study discusses the feasibility of such multiphase softsensors. In particular, the required and sufficient set of measurements is defined. Furthermore, the influence of model error and measurement noise on the quality of estimates is studied.

This paper is organized as follows. First, the pipe flow model and the computational setup for soft-sensing are given. Next, the description of the used soft-sensing algorithm is presented. Finally, the simulations results are given.

\section{DYNAMIC FLOW MODEL}

A model describing one-dimensional two-phase flow in pipes consists of non-linear partial differential equations describing mass and momentum conservation for each phase. This model is obtained from cross-sectional averaging of the Navier-Stokes equations and replacing diffusion terms by empirical correlations. Since the main purpose of this work is the application of estimation techniques, no detailed flow description is required. Therefore, it was assumed that the gas and liquid are travelling with the same velocity $u$ (Vicente, et al., 2001).

The simplified mass conservation equations are

$$
\begin{aligned}
& \frac{\partial}{\partial t}\left(\rho_{l} H\right)+\frac{\partial}{\partial s}\left(\rho_{l} H u\right)=\Phi_{l} \\
& \frac{\partial}{\partial t}\left(\rho_{g}(1-H)\right)+\frac{\partial}{\partial s}\left(\rho_{g}(1-H) u\right)=\Phi_{g}
\end{aligned}
$$

Where $H$ is the liquid volume fraction, $\rho_{g}$ is the gas density, $\rho_{l}$ is the liquid density, $t$ denotes time and $s$ denotes the coordinate along the length of the pipe. $\Phi_{l}$ and $\Phi_{g}$ are the mass sources representing the inflow from a reservoir to the pipe. These sources are normally time dependent.

Although the continuity equations have been written for each phase it is common to write the momentum equation for the mixture.

$$
\frac{\partial}{\partial t}\left(\rho_{m} u\right)+\frac{\partial}{\partial s}\left(\rho_{m} u^{2}\right)=-\frac{\partial p}{\partial s}-S_{f r}
$$

Where $\rho_{m}$ is the mixture density defined by

$$
\rho_{m}=\rho_{g}(1-H)+\rho_{l} H
$$

A frequently used model for frictional losses in the momentum equation has the form

$$
S_{f r}=\frac{\lambda}{2 d} \rho_{m} u^{2}
$$

Here $d$ is the pipe diameter and $\lambda$ is the friction factor, which is a function of the Reynolds number and pipe roughness $k$. In this study the Techo formula is used: $\lambda=\left[-0.8685 \ln \left(\frac{1.964 \ln (\mathrm{Re})-3.8215}{\operatorname{Re}}+\frac{k}{d \cdot 3.71}\right)\right]^{-2}$

Here Re is the Reynolds number defined as

$\operatorname{Re}=u d \rho_{m} / \mu_{m}$

with the mixture viscosity $\mu_{m}$ calculated in terms of liquid volume fraction and gas $\mu_{g}$ and liquid $\mu_{l}$ viscosities

$\mu_{m}=\mu_{g}(1-H)+\mu_{l} H$

The gas is treated as a compressible phase with a corresponding equation of state given in the form

$\rho_{g}=f(p)$

The closure of the problem is given by the following boundary conditions.

$$
\begin{aligned}
& p(s=L, t)=p_{\text {out }}, p(s, t=0)=p_{\text {out }} \\
& u(s=0, t)=u_{\mathrm{inf}}, u(s, t=0)=u_{\mathrm{inf}} \\
& H(s=0, t)=H_{\mathrm{inf}}, H(s, t=0)=H_{\mathrm{inf}}
\end{aligned}
$$

Here the subscripts inf and out refer to inflow and outflow cross-section of the pipe respectively. $L$ denotes the length of the pipe.

\section{DATA ASSIMILATION}

\subsection{State-space form of the model equations}

Due to the nonlinearity of the given equation system (1)-(12) the numerical solution is needed in order to solve it for the dependent variables. For the discretization of the simulation domain a staggered grid approach has been used, meaning that the different grids are used for the continuity and momentum equation. Afterwards, the governing equations are integrated over different control volumes. Any solution procedure can be applied for solving the non-linear system of algebraic equations. Finally, the model can be written in the following state-space notation (Crassidis, 2004):

$x_{k}=f\left(x_{k-1}, u_{k-1}\right)$

Here $u_{k-1}$ is the model input representing the inflow from reservoir to wellbore. $x_{k-1}$ is the state vector evaluated on the previous time step. Using the primitive set of variables, the state vector can be written as

$$
x=[p u H]^{T}
$$

Here $p, u$ and $\mathrm{H}$ are the vectors, representing pressure, velocity and liquid volume fraction related to the spatial grid.

\subsection{Formulation of the inverse problem}

The computational setup for the inverse problem is shown in Figure 2. It should be noted here, that only the horizontal part of the well is being modelled, and the outflow measurements are assumed to be available directly at the outflow cross-section of the horizontal part. 


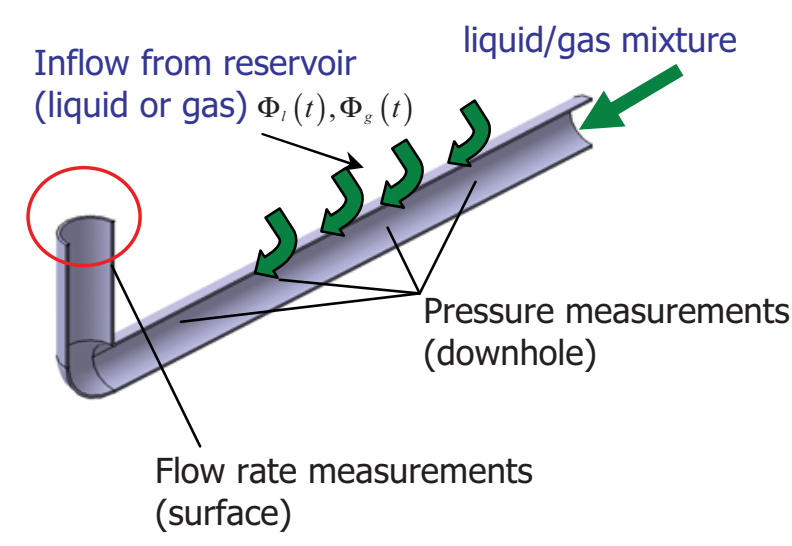

Fig. 2. Scheme of the computational setup for soft-sensing.

For the soft sensing purposes the augmented state vector is introduced:

$$
X=\left[p_{i} u_{i} H_{i} \mid \Phi_{g i} \Phi_{l i}\right]^{T}
$$

Here $i$ indicates the number of the cell defined by the numerical discretization.

It is assumed that several downhole pressure measurements are available. Moreover, outflow information about flow rates is also known, giving the following measurement vector:

$$
y=\left[p_{i} u_{\text {out }} H_{\text {out }}\right]^{T}
$$

Finally, the data assimilation problem can be formulated as follows: with the measurements (16) and the flow model (1)(12) available the components of the augmented state vector should be estimated.

Due to a lack of experimental data, a set of synthetic measurements has been used as a source for soft-sensing. First, a twin experiment concept has been implemented. Here the same mathematical model was used both for generating measurements with predefined inflow distribution and the inverse modelling, when missing dynamic variables are estimated by means of the soft-sensing algorithm. In order to mimic the situation of testing the soft-sensor with "real-life" data, simulation results from the commercially available flow simulator OLGA were used in the second test case.

\subsection{Ensemble Kalman filtering}

One way to solve estimation problems via the sequential data assimilation algorithm is by using the Kalman filter equations. The Kalman filter is a stochastic recursive estimator, which estimates the values of model states and unknown input by integrating measured data in a mathematical model in real-time. Due to its straightforward numerical implementation and recursive nature, the Kalman filter algorithm is very well adapted to online model calibration.

Kalman filtering was initially developed for linear dynamic systems. Although several extensions of the Kalman filter exist for non-linear system, here the ensemble Kalman filter (EnKF) is used (Evensen, 1994). In this approach, the approximation of the error covariance matrix is calculated using an ensemble of possible model realizations, which are propagated according to the full dynamics of the system.

In order to initialize the filter the initial ensemble is generated. Here a mean value of the initial augmented state vector $\bar{X}_{0}^{a}$ and a corresponding covariance matrix $Q_{0}$ is required. The mean value of the initial ensemble should be a good estimate of the true initial state. The members of the ensemble are generated randomly according to a Gaussian distribution. The $j$ 'th member of the ensemble is defined as

$X_{0, j}^{a}=\bar{X}_{0}^{a}+w_{0, j}$

With an EnKF the augmented state vector, which also contains the inflow input, is estimated in a recursive manner through the following two steps:

1) The forecast step, which consists in running the flow model one time step forward for each member of the ensemble. This leads to

$X_{k, j}^{f}=f\left(X_{k-1, j}^{a}\right)+w_{k, j}$

Here $w_{k, j}$ is a Gaussian zero mean white noise with the corresponding covariance matrix $Q_{\mathrm{k}}$ representing the model error. This noise is only added to components of the state vector, which produce the most uncertainty in a simulation. These are in this case the inflow sources $\Phi_{l}$ and $\Phi_{g}$.

Using the calculated forecast of ensemble states, the error covariance matrix can be calculated using the covariance matrix of the ensemble. The mean value of the ensemble is given by

$$
\bar{X}_{k}^{f}=\frac{1}{N} \sum_{j=1}^{N} X_{k, j}^{f}
$$

And the error covariance matrix is then calculated as

$P_{k}^{f}=L_{k}^{f}\left(L_{k}^{f}\right)^{T}$

With

$L_{k}^{f}=\frac{1}{\sqrt{N-1}}\left[\left(X_{k, 1}^{f}-\bar{X}_{k}^{f}\right)\left(X_{k, 2}^{f}-\bar{X}_{k}^{f}\right) \ldots\left(X_{k, N}^{f}-\bar{X}_{k}^{f}\right)\right]$

where $N$ is the number of members in the ensemble.

2) The analysis step, which takes into account measurements. The errors in the measurements are assumed to be statistically independent with known variances. This leads to a diagonal covariance matrix for the measurement errors. As it has been pointed out in Burgers et al. (1998), it is necessary to define new measurements for the proper error propagation. Therefore, a new observation vector is introduced for each member of the ensemble

$y_{k, j}=M_{k} \cdot X_{k, j}+v_{k, j}$

Here $M_{k}$ is the measurement matrix and $v_{k, j}$ is the measurement noise generated from a normal distribution with zero mean and covariance matrix $R_{k}$. 
The Kalman gain is then calculated as follows

$$
K_{k}=P_{k}^{f} M_{k}^{T}\left(M_{k} P_{k}^{f} M_{k}^{T}+R_{k}\right)^{-1}
$$

The analyzed state for each member of the ensemble is given by

$$
X_{k, j}^{a}=X_{k, j}^{f}+K_{k}\left(y_{k, j}-M_{k} X_{k, j}^{f}\right)
$$

The mean value of the analyzed ensemble is

$$
\bar{X}_{k}^{a}=\frac{1}{N} \sum_{j=1}^{N} X_{k, j}^{a}
$$

The unknown inflow sources are updated at each time step measurements are available and extracted from the augmented state vector. The analyzed error covariance matrix, from which the estimation error of the inflow parameters can be defined, is then approximated by

$$
P_{k}^{a}=\left(I-K_{k} M_{k}\right) P_{k}^{f}
$$

An important issue with the use of the EnKF is the size of the ensemble. Based on the experience of data assimilation for large-scale atmospheric models (Houtekamer and Mitchell, 1998), 100 ensemble members have been chosen for the ensemble Kalman filtering. The optimal size of the ensemble is, however, not known and it is a subject for future research.

\section{RESULTS AND DISCUSSIONS}

\subsection{Soft sensing under measurement error}

A first test case considered uses a twin experiment concept. Here the same mathematical model is used for generating the measurements with predefined inflow distribution. This study deals with two-phase liquid/gas flow and the details of the initial data are given in Table 1 . The sketch of the simulation domain is given in Figure 3. The inflow profiles are given only as a reference since they are unknown and have to be estimated via the proposed data assimilation procedure.

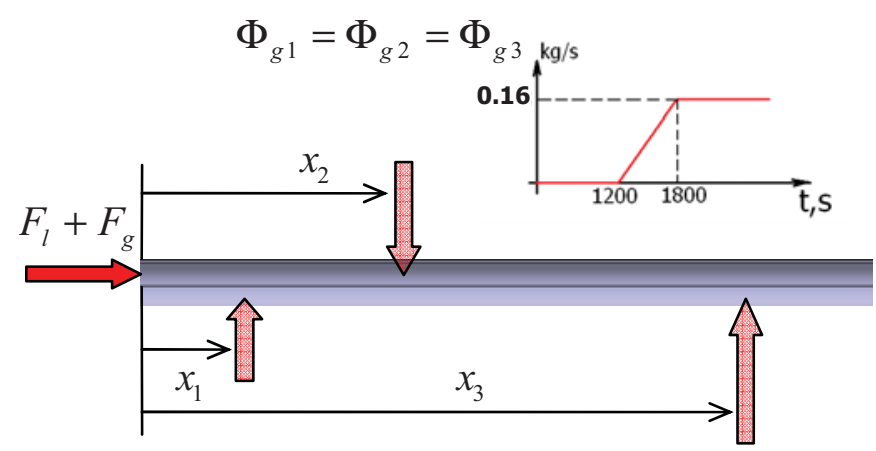

Fig. 3. Computational setup for soft-sensing.

Initially the well produces a mixture of liquid and gas with a total flow rate of $10 \mathrm{~kg} / \mathrm{s}$. After 20 minutes of production, gas is injected in three locations of the wellbore. The amount of gas injected increases linearly up to $0.5 \mathrm{~kg} / \mathrm{s}$ during next 30 minutes and afterwards kept constant for the last 10 minutes of simulation.
The soft-sensor has been tested using the following measurement layout. The number of pressure measurements was taken equal to number of grid nodes obtained from the discretization. The velocity and liquid volume fraction measurements are located at the last grid block of the simulation domain.

Table 1. Initial data for the numerical experiments

\begin{tabular}{|c|c|}
\hline Quantity & Value \\
\hline Pipe diameter, $\mathrm{m}$ & 0.05 \\
\hline Pipe length, $\mathrm{m}$ & 100 \\
\hline Liquid density, $\mathrm{kg} / \mathrm{m}^{3}$ & 1000 \\
\hline Liquid viscosity, Pa's & 0.001 \\
\hline Gas reference density, $\mathrm{kg} / \mathrm{m}^{3}$ & 118.9 \\
\hline Gas viscosity, $\mathrm{Pa}$ s & $1.82 \cdot 10^{-5}$ \\
\hline Time step, $\mathrm{s}$ & 60 \\
\hline Inflow liquid rate $F_{l}, \mathrm{~kg} / \mathrm{s}$ & 9.5 \\
\hline Inflow gas rate $F_{g}, \mathrm{~kg} / \mathrm{s}$ & 0.5 \\
\hline $\mathrm{x}_{1}, \mathrm{~m}$ & 15 \\
\hline $\mathrm{x}_{2}, \mathrm{~m}$ & 45 \\
\hline $\mathrm{x}_{3}, \mathrm{~m}$ & 75 \\
\hline Absolute roughness, $\mathrm{m}$ & 0 \\
\hline Number of grid nodes & 12 \\
\hline
\end{tabular}

The Kalman filter initialization is based here on the outflow values of velocity and liquid volume fraction, which are assumed to be known from a flow meter. Since all the pressure measurements are available, pressure is initialized from the current pressure distribution. The synthetic measurements representing downhole pressure and liquid outflow flow rate are generated using equations (1)-(12). A zero mean white Gaussian noise is then added to mimic the uncertainty in measurements.

Table 2. Measurement noise used in simulations

\begin{tabular}{|c|c|}
\hline Uncertainty in pressure measurements & $0.5 \%$ \\
\hline $\begin{array}{c}\text { Uncertainty in outflow velocity } \\
\text { measurements }\end{array}$ & $1 \%$ \\
\hline $\begin{array}{c}\text { Uncertainty in liquid volume fraction } \\
\text { measurements }\end{array}$ & $1 \%$ \\
\hline
\end{tabular}

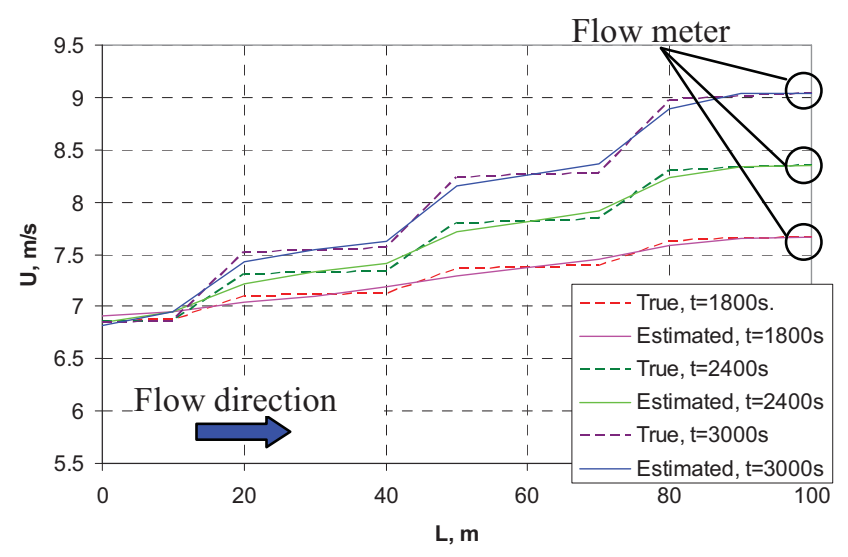

Fig. 4. Comparison of estimated and true flow velocity. 
The results of the simulation are given in Figures 4-5. Figure 4 shows the comparison between the estimated and true velocity distributions along the pipe length. Flow velocity is used to allocate the zones where a fluid is entering or leaving the wellbore. In order to identify the type of fluid, the distribution of the estimated liquid volume fraction is required. It is depicted in Figure 5. The results are given for three time instants 30 minutes, 40 minutes and 50 minutes. Since the pressure is available continuously from the measurements it is not depicted as a soft-sensing result.

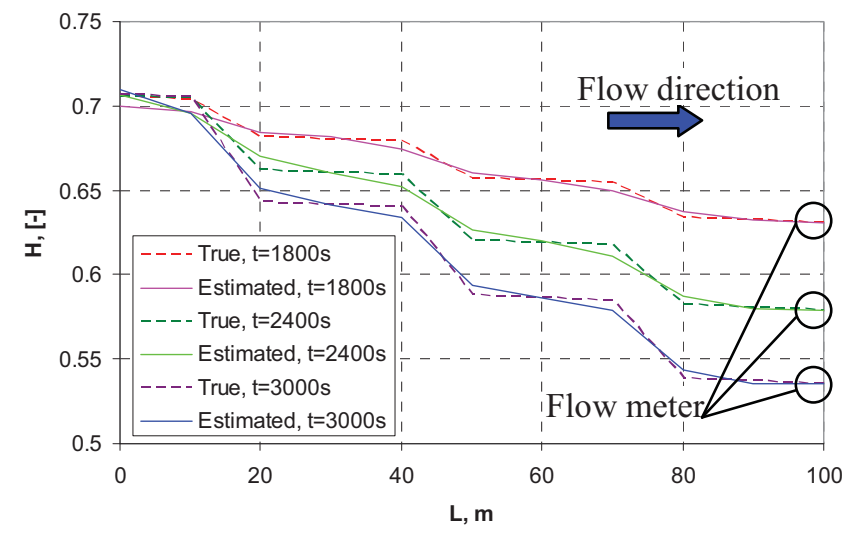

Fig. 5. Comparison of estimated and true liquid fraction.

The results show that the proposed soft-sensor, for the given simplified setup, is very well capable of reproducing the flow rate and liquid volume fraction distributions along the considered well part, even when measured data contains a certain measurement error. Therefore, it is capable to detect multiple fluid sources as it is depicted in the figures.

\subsection{Soft sensing under model error}

The second study provides an assessment of the influence of the model error on the soft-sensing estimation results. A similar soft-sensing setup was used as depicted in Figure 2 for case study 1. An important difference, however, was that the "true" well was not the same as the model used in the soft-sensor. The true wellbore measurements were obtained from the commercially available simulator OLGA. This was done to assess the inevitable effect of the model error on the soft-sensing estimation results. Here both transient gas and liquid sources are present in a computational setup. Liquid is injected in the first part of the pipe, while a gas source is present close to its outflow cross-section. This situation is a rough approximation of the gas breakthrough scenario. The scheme of the simulation domain is given in Figure 6.

Due to differences between the flow model used in OLGA simulator and the soft-sensor developed, one can point at the following sources of the model error:

- Friction factor correlation

- Fluid properties

- Simulation grid

- Mathematical model

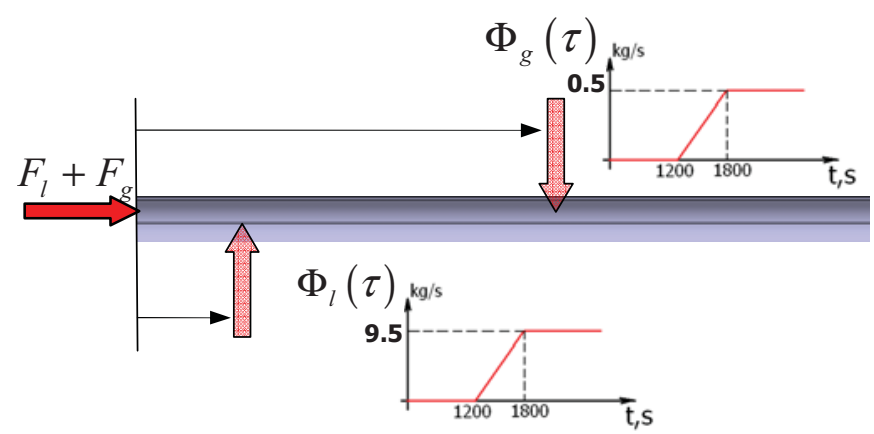

Fig. 6. Computational setup for soft-sensing. Test case 2.

A particularly important modelling assumption for performing OLGA simulations was to keep a dispersed bubble flow regime, since the model used for soft-sensing is valid only for that type of multiphase flow. This was possible using the same set of input parameters, as for the test case 1 . The OLGA simulations were performed with 10 grid nodes, where the source term for liquid has been defined in the third grid block, and for gas in the eighth grid block. This consequently led to a soft-sensing setup with 10 available pressure measurements.

Figures 7 and 8 represent the estimated flow velocity and liquid volume fraction.

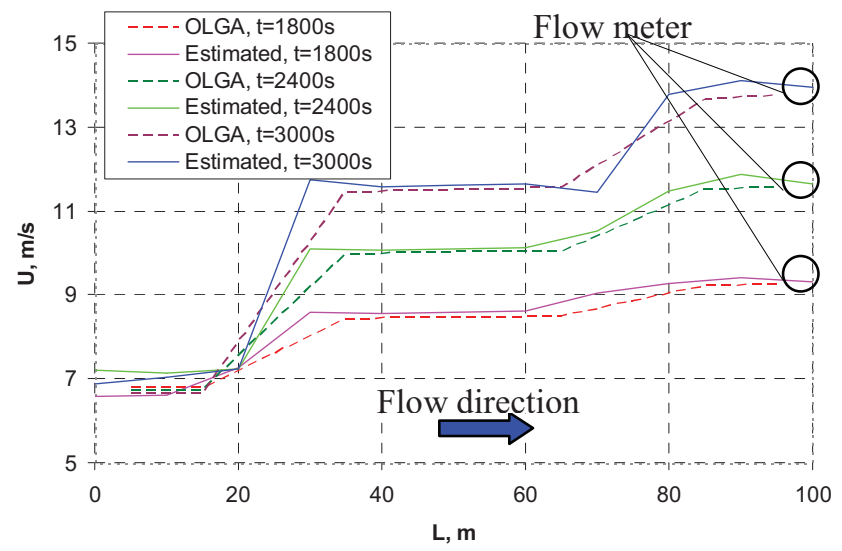

Fig. 7. Estimated velocity profile for the OLGA data.

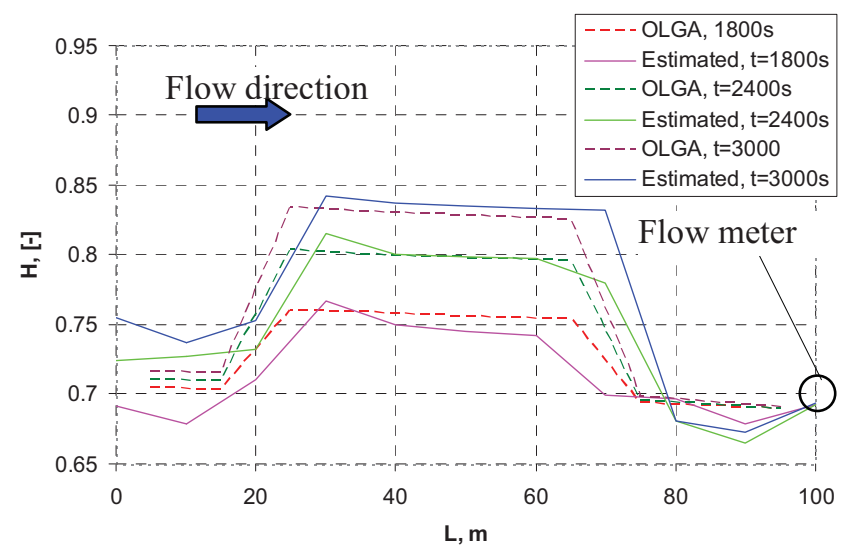

Fig. 8. Estimated liquid fraction profile for the OLGA data. 
The results obtained under model error are not as accurate as for the twin-experiment. However, it is still possible to allocate easily zones of liquid or gas inflow. A displacement of the estimated profiles with respect to the true ones is observed. This can be explained by the use of a different grid in the OLGA simulator and different interpolation of the flow variables between grid nodes and edges.

\section{SUMMARY AND CONCLUSIONS}

By means of two case studies, some limitations and possibilities of soft-sensor multiphase flow meters have been studied. The proposed soft-sensor is based on the ensemble Kalman filter approach and requires as the input the dynamic model of the pipe flow together with pressure measurements available downhole and one composition and velocity measurement at the outflow.

It has been shown, that for a two-phase flow formulation it is possible to reconstruct the distributions of the flow velocity and liquid volume fraction along a pipe and to allocate the inflow of certain fluids in a specific location along it.

The results indicate that the proposed method is quite stable for a certain range of wellbore operational conditions, and capable of taking into account measurement and model error.

\section{ACKNOWLEDGEMENTS}

This work has been supported by ISAPP knowledge center, which is a joint research project of Shell, TNO and Delft University of Technology. The authors would also like to thank Wouter Schiferli (TNO Science and Industry) for performing the OLGA runs.

\section{REFERENCES}

Bloemen, H.H.J., Belfroid, S.P.C., Sturm, W.L., and Verhelst, F.J.P.C.M.G. (2004). Soft Sensing for Gas-Lift Wells. SPE paper 90370, Proc. SPE Annual Technical Conference and Exhibition, Houston, Texas, USA.

Burgers, G., van Leeuwen, P.J., and Evensen, G. (1998) On the analysis scheme in the ensemble Kalman Filter. Monthly Weather review. Vol. 126, 1719-1734.

Crassidis J.L. and Junkins J.L. (2004). Optimal estimation of Dynamic systems. Chapman \& Hall/CRC. Florida.

De Kruif, B., Leskens, M., van der Linden, R., Alberts, G., and Smeulers, J. (2008) Soft-sensing for multilateral wells with downhole pressure and temperature measurements. SPE paper 118171, Proc. Dhabi International Petroleum Exhibition and Conference, Abu Dhabi, UAE,

Evensen, G. (1994). Sequential data assimilation with a nonlinear quasi-geostrophic model using Monte Carlo methods to forecast error statistics," J. Geophys. Res., Vol. 99(C5), 10143-10162.

Evensen, G., Hove J., Meisingset, H.C., Reiso E., Seim, K.S., and Espelid, O. (2007). Using the EnKF for Assisted History Matching of a North Sea Reservoir Model. SPE paper 106184, Proc. SPE Reservoir simulation symposium, Woodlands, Texas, USA.
Glandt, C.A. (2003). Reservoir aspects of smart wells. SPE paper 81107, Proc. SPE Latin American and Caribbean Petroleum Engineering Conference, Trinidad, West Indies.

Houtekamer, P.L., Mitchell, H.L., (1998). Data assimilation using an ensemble Kalman filter technique. Monthly Weather review. Vol. 126, 796-911.

Jansen J.D., Bosgra O.H., and Van den Hof P.M.J. (2008). Model-based control of multiphase flow in subsurface oil reservoirs. Journal of Process Control, Vol. 18, 846-855.

Jazwinski, A. H. (1970). Stochastic Processes and Filtering Theory. Academic Press, New York.

Julier, S., Uhlmann, J., and Durrant-Whyte, H.F. (2000). A new method for the non-linear transformation of means and covariances in filters and estimators. IEEE Transcations on Automatic Control. Vol. 45(3), pp. 477 482.

Kalman, R.E. (1960). A new approach to linear filter and prediction theory. Trans. ASME, Series D, Journal of Basic Engineering, Vol. 82, pp. 35 - 45.

Leskens, M., de Kruif, B., Belfroid, S., Gryzlov, A., and Smeulers, J. (2008). Downhole Multiphase Metering in Wells by Means of Soft-Sensing. SPE paper 112046, proc. SPE Intelligent Energy Conference, Amsterdam, The Netherlands.

Lorentzen, R.J., Fjelde, K.K.,Frøyen J, Lage A. C. V. M., Nævdal G., and Vefring E.H. (2001). Underbalanced and Low-head Drilling Operations: Real Time Interpretation of Measured Data and Operational Support, Proc. SPE Annual Technical Conference and Exhibition, SPE 71384, New Orleans, Louisiana.

Lorentzen, R.J., Nævdal, G., and Lage, A.C.V.M. (2003). Tuning of parameters in a two-phase flow model using an ensemble Kalman filter. International Journal of Multiphase Flow. Vol. 29(8), pp. 1283-1309.

Nævdal, G., Johnsen, L.M., Aanonsen, S.I., and Vefring, E.H. (2003). Reservoir Monitoring and Continuous Model Updating Using Ensemble Kalman Filter. SPE paper 84372, Proc. SPE Annual Technical Conference and Exhibition, Denver, Colorado, USA.

Vicente, R., Sarica, C., and Ertekin, T. (2001) .A Two-Phase Model Coupling Reservoir and Horizontal Well Flow Dynamics. SPE paper 69570, Proc. SPE Latin American and Caribbean Petroleum Engineering Conference, Buenos Aires, Argentina. 